
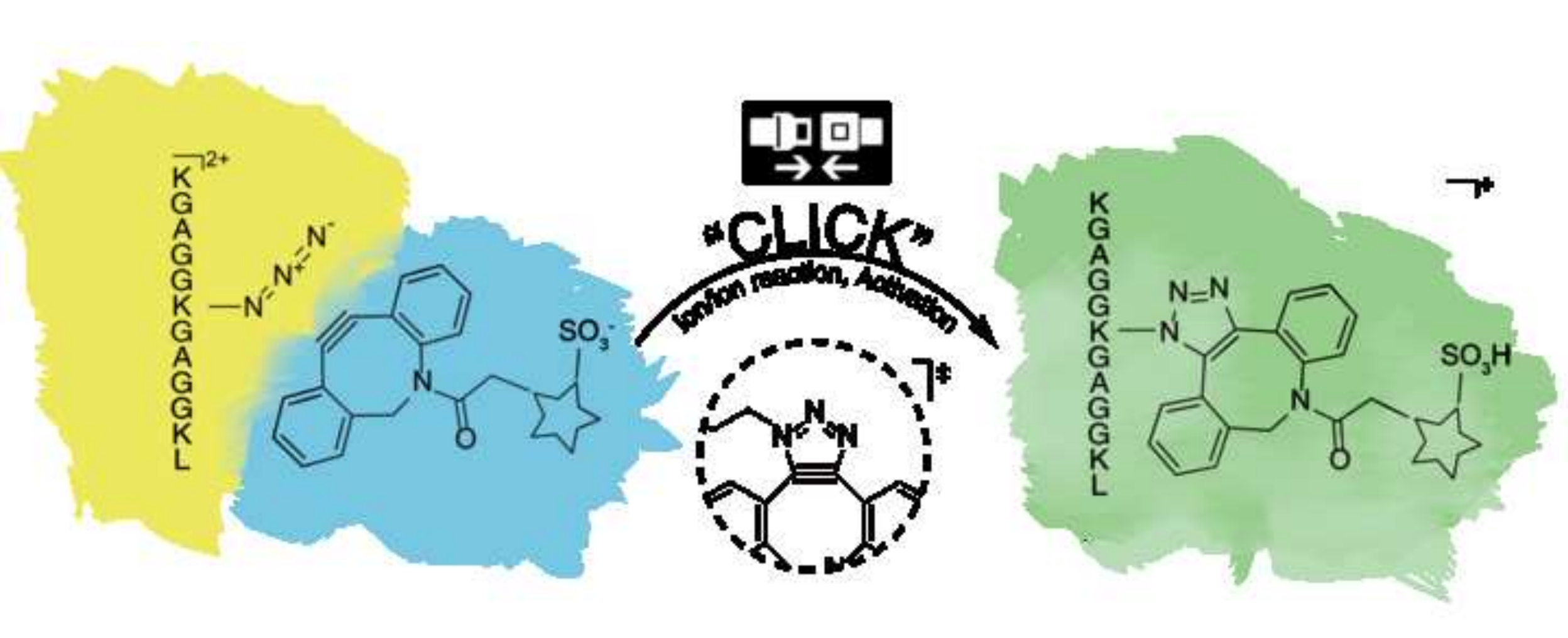


\title{
Gas Phase Click Chemistry via Ion/Ion Reactions
}

\author{
Jiexun Bu, Alice L. Pilo, Scott A. McLuckey* \\ Department of Chemistry \\ Purdue University \\ West Lafayette, IN 47907-2084
}

Running title: Gas-phase Ion Click Chemistry

*Address reprint requests to:

Dr. S. A. McLuckey

560 Oval Drive

Department of Chemistry

Purdue University

West Lafayette, IN 47907-2084, USA

Phone: (765) 494-5270

Fax: (765) 494-0239

E-mail: mcluckey@purdue.edu 


\section{ABSTRACT}

The 1,3-dipolar cycloaddition between azides and alkynes, i.e., 'click' chemistry, has been demonstrated in the gas phase via ion/ion reactions. Doubly protonated azidemodified peptide cations were reacted with singly deprotonated dibenzoazacyclooctyne DIBAC reagent anions to form a long-lived complex monocation. Similarly, ion/ion complex monoanions were generated in the negative mode via reactions between singly protonated azide-containing dications and doubly deprotonated DIBAC reagent anions. Activation of ion/ion complexes in either polarity resulted in the formation of a triazole, a covalent linkage that can be further probed via $\mathrm{MS}^{n}$. The click chemistry coupled species synthesized in solution and subsequently transferred into the gas phase yielded the same products as those synthesized in gas phase when activated in the mass spectrometer. Analogous click chemistry products were synthesized in the solution phase and activated via tandem mass spectrometry as a control for the dissociation of the novel gas-phase reaction products. Density functional theory (DFT) calculations were performed to provide estimates for key processes (e.g., reaction barriers and energies) that can occur in the overall reaction. The work herein demonstrates that it is possible to conjugate azide and alkyne functional groups in the gas-phase, and represents another example of the growing number of selective covalent reactions that can take place via gas-phase ion/ion reactions.

Key words: Ion/ion reactions, click chemistry, azide reactivity, gas-phase cycloaddition 


\section{INTRODUCTION}

Electrospray ionization (ESI) was introduced [1] in the 1960s and implemented with mass spectrometry developed [2] in the 1980s as a soft ionization method. It has since become one of the most frequently used ionization methods in many applications of mass spectrometry, particularly for liquid chromatography-mass spectrometry (LC-MS) [3] and biomolecule analysis [2]. In order to facilitate mass spectral analyses using ESI, solution phase derivatization strategies are commonly employed to aid in specific aims such as quantification [4,5], structural characterization [6,7], and signal enhancement [8]. Some of these modifications can also be performed in the gas phase via ion/ion $[9,10]$ or ion/molecule reactions [11].

Gas phase ion/ion reactions have proven to be capable of effecting a wide range of ion transformations from ion type to another within a mass spectrometer. Typically these reactions fall into the categories of small particle transfer and covalent modification. Small charged particle transfers, such as proton transfer and electron transfer $[9,10]$ can occur either at crossing points on the potential energy surface $[9,12]$ or through the formation of a long-lived electrostatic complex. Electron transfer from a reagent anion to a multiply protonated peptide or protein is the first step in the electron transfer dissociation (ETD) process, which has been widely used as a structural probe [13], while proton transfer has been shown to be particularly useful in the manipulation of protein charge states [14]. Ion/ion reactions that involve covalent bond formation proceed exclusively via formation of a long-lived complex [15]. An example of covalent modification in the gas phase is Schiff base formation between formyl-benzenesulfonic acids and primary amine groups on peptides [ 16 ]. Reactions between N- 
hydroxysuccinimide esters and a nucleophile, such as a primary amine [17], a guanidine group [18], and a carboxylate [19], have also been described. The covalent labeling of carboxylic acid groups was achieved via ion/ion reactions with carbodiimide reagents [20]. Additional examples of covalent modification in the gas phase via ion/ion reactions include the oxidation of polypeptides with periodate anion [21] and persulfate [22] derivatives and the alkyl transfer reaction from ammonium and sulfonium cations to various anionic sites, such as carboxylates, sulfonates, and phosphates [23]. Here we present the gas phase 1,3-dipolar cycloaddition between azide and alkyne functionalities, commonly referred to as 'click chemistry' [24].

Uncatalyzed azide-alkyne 1,3-dipolar cycloaddition was first discovered in 1963 by Huisgen [25]. The reaction described by Huisgen required high pressures and temperatures and was not widely employed until Rostovtsev et al. and Tornoe et al. developed the copper(I) catalyzed azide-alkyne cycloaddition, which is widely used in materials and biology applications $[26,27]$. The term "click chemistry" was first defined by Sharpless et al. in 2001 as chemistry that could quickly and reliably connect small units together and meets a series of requirements articulated in the original [24]. In 2007, Baskin et al. developed the first copper-free click chemistry reaction using a fluorinated cyclooctyne-based reagent [28]. Many copper-free click chemistry reagents have since been developed, including difluorinated cyclooctyne (DIFO) [28], dibenzocyclooctyne (DIBO) [29 ], dibenzoazacyclooctyne (DIBAC) [30 ], and biarylazacyclooctynone (BARAC) [31]. Click chemistry has found a niche in synthetic chemistry as it provides methods of connecting multiple building blocks together with high specificity and efficiency under relatively mild conditions. Examples include the conjugation of 
biopolymers and dyes, two different active groups in a drug, etc. [32]. Currently, click chemistry plays important roles in diverse areas including drug discovery, materials science, and bioimaging. [32] Here, we describe a novel gas phase approach for azidealkyne click chemistry via ion/ion reactions. This method adds another tool for gas phase covalent modification, which might be used in various applications such as site specific probing and labeling, the location of olefins, or cross-linking. Applications of gas phase click chemistry are not emphasized here. Rather, we demonstrate the viability of gas phase click chemistry and provide some insights into the energetics associated with the reaction.

\section{Experimental}

Materials. Methanol was purchased from Mallinckrodt (Phillipsburg, NJ, USA). HPLC grade water was purchased from Fisher Scientific (Waltham, MA). Rhodamine 110-azide, dibenzoazacyclooctyne-N-hydroxysuccinimidyl ester (DIBAC-NHS), sulfanilic acid, potassium 2-iodo-5methylbenzenesulfonate, and 2-amino-1,5naphthalenedisulfonic acid were purchased from Sigma-Aldrich (St. Louis, MO). The peptide KGAGGKGAGGKL was custom synthesized by NeoBioLab (Cambridge, MA). Azidobutyric acid NHS ester was purchased from Lumiprobe (Hallandale Beach, FL). All reactant and reagent ions were generated via nano-electrospray from 1:1 (v/v) water:methanol solutions.

Solution Phase Synthesis. The deprotonated DIBAC reagents were synthesized in the solution phase by taking advantage of N-hydroxysuccinimide (NHS) chemistry [33]. A $5 \mathrm{mM}$ solution of DIBAC-NHS (Figure 1(a)) in DMF was reacted with one equivalent of sulfanilic acid. The reaction was carried out at $4{ }^{\circ} \mathrm{C}$ overnight. The product 
(Figure 1(b)) is referred to as DIBAC-sulfonate. Similarly, DIBAC-NHS was reacted with 2-amino-1,5-naphthalenedisulfonic acid under the same conditions to form a doubly deprotonated reagent (Figure 1(c)), referred to as DIBAC-disulfonate. Both product solutions were diluted 10-fold with 1:1 (v/v) water:methanol.

(a)<smiles>O=C(CCC(=O)N1Cc2ccccc2C#Cc2ccccc21)ON1C(=O)CCC1=O</smiles>

(b)

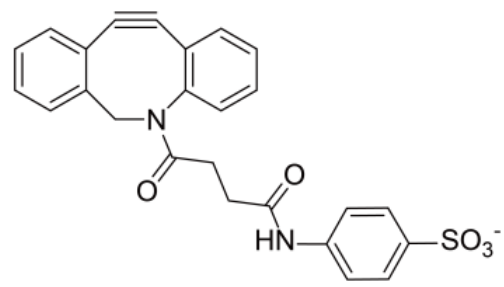

(d) (c)<smiles>CN(N)N</smiles>

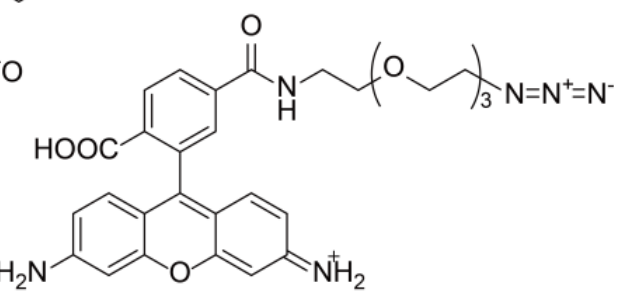

Figure 1. Structures of (a) DIBAC-NHS, (b) DIBAC-sulfonate, (c) DIBAC-disulfonate, and (d) Rhodamine 110-azide.

The peptide KGAGGKGAGGKL was reacted with equimolar azidobutyric NHS ester at $4{ }^{\circ} \mathrm{C}$ in $1: 1$ (v:v) water:methanol overnight. The resulting solution generated doubly protonated, azide-modified KGAGGKGAGGKL, referred to as azide-K.K.KL, upon positive nanoelectrospray ionization (nESI). Activation of the protonated azideK.K.KL (Figure S1(a)) indicated that the azide attached to an amine on one of the lysine side-chains or the $\mathrm{N}$-terminus in a non-selective fashion. 
Solution phase coupled click chemistry products were synthesized by combining equimolar amounts of the analyte and the reagent in a 1:1 (v/v) water:methanol solution for over 30 minutes at room temperature. The analyte and reagent pairs were DIBACsulfonate/azide-K.K.KL and DIBAC-disulfonate/Rhodamine 110-azide (Rhodamine 110azide structure shown in Figure 1(d)).

Mass spectrometry. All experiments were performed using a QTRAP 4000 hybrid triple quadrupole/linear ion trap (AB SCIEX, Concord, ON, Canada), previously modified for ion/ion reactions [34]. Anions and cations were sequentially injected into the instrument via alternately pulsed nESI [35] and isolated in Q1 before being transferred into the $\mathrm{q} 2$ reaction cell, which has been modified to allow for mutual storage of both ion polarities [36]. In the $\mathrm{q} 2$ reaction cell, the ions were allowed to react for a defined period of time ranging from 100-500 ms. Reaction products are then transferred to Q3, where they are further probed via $\mathrm{MS}^{n}$ and mass analyzed using mass-selective axial ejection (MSAE) [34,37].

Density Functional Theory (DFT) Calculations. DFT calculations were used to characterize reaction pathways. All structure optimizations and zero-point corrected energies (ZPE) were calculated using the Gaussian 09 package [38] at the unrestricted B3LYP/6-311G++(d,p) level of theory. Transition states (TS) were searched for using QST3 options and confirmed with intrinsic reaction coordinate (IRC) calculations [39]. All stationary points have been identified as local minima (zero imaginary frequencies) or transition states (one imaginary frequency). 


\section{RESULTS AND DISCUSSION}

Proteins, peptides and azide-containing dyes are common substrates that appear in biological click chemistry applications. Here, a solution phase modified azide-containing peptide and Rhodamine-110-azide were used as model analytes. DIBAC was selected as the reagent for click chemistry ion/ion reactions due to its high reactivity in the absence of a copper catalyst. NHS chemistry [33] was used in the solution phase to modify DIBAC to form a singly deprotonated reagent, DIBAC-sulfonate, and a doubly deprotonated DIBAC reagent, DIBAC-disulfonate. The sulfonates serve as strongly interacting groups with protonated sites to form relatively stable electrostatic interactions between the analyte and reagent, which aids in the formation of a long-lived complex. Analyte ions were stored in the presence of oppositely charged DIBAC reagent ions in the gas phase. The analyte-reagent complexes formed via cation-anion attachment were then subjected to collision-induced dissociation (CID) for structural interrogation. There is no signature mass loss for a cycloaddition reaction (Scheme 1). Therefore, evidence for covalent bond formation is derived from cleavages of analyte covalent bonds with retention of the covalent modification

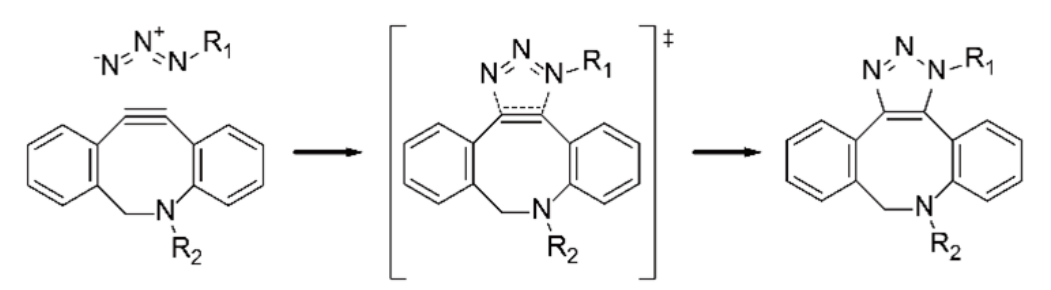

Scheme 1 - 1,3-dipolar cycloaddition. 
The cycloaddition chemistry was first noted with the electrostatic complex generated in the positive polarity. The peptide KGAGGKGAGGKL (abbreviated as K.K.KL) was modified with an azide (indicated as azide-K.K.KL) and the doubly protonated species of K.K.KL $(\mathrm{m} / z$ 500.8) and azide-K.K.KL ( $\mathrm{m} / z$ 556.3) were simultaneously subjected to ion/ion reactions with singly deprotonated DIBAC-sulfonate reagent anions in the $\mathrm{q} 2$ collision cell (Figure 2(a)). The major signals present in Figure 2(a) correspond to residual unreacted analyte ions (i.e., $[\mathrm{K} . \mathrm{K} . \mathrm{KL}+2 \mathrm{H}]^{2+}$ and $[$ azideK.K.KL+2H] $\left.]^{2+}\right)$ proton transfer products (i.e., $[\text { K.K.KL }+\mathrm{H}]^{+}(\mathrm{m} / \mathrm{z}$ 1000.5) and [azideK.K.KL+H] $\left.{ }^{+}(m / z, 1111.5)\right)$ and the [azide-K.K.KL+2H/DIBAC-sulfonate] ${ }^{+}(m / z, 1571.6)$ cation-anion complex (indicated as 'complex' in Figure 2(a)). Activation of the low abundance [K.K.KL+2H/DIBAC-sufonate $]^{+}$complex, which is unlabeled in Figure 2(a), gives rise exclusively to the proton transfer product, viz., singly protonated K.K.KL (Figure S1(b)). Upon isolation and CID of the [azide-K.K.KL/DIBAC-sufonate+H] ${ }^{+}$ complex, products from proton transfer, viz., singly protonated azide-K.K.KL, and backbone fragmentation were observed (Figure 2(b)). Both modified (shown in red) and unmodified fragments were produced from backbone fragmentation. It is worth noting that the mass of the modification on all modified fragments corresponded to the product expected from cycloaddition. No azide-only modifications were observed. This is consistent with the backbone fragments arising from a population of analyte ions that underwent cycloaddition and the proton transfer product ions stemming from a population of ions in which no covalent chemistry took place. For the experiment leading 
to Figure 2(b), proton transfer gave rise to $23 \%$ of the product ion signal while backbone fragmentation gave rise to $77 \%$.

The interpretation provided above assumes that proton transfer and backbone cleavage arise from different non-interconverting structures (i.e., one in which a covalent reaction has occurred and one in which no cycloaddition occurred). An alternative interpretation might assume that proton transfer and covalent bond cleavage are competitive processes from a population of complex ions with a single structure. If the ion/ion complex population is comprised of a mixture of structures, it might be possible to alter the relative composition of the structures by subjecting them to a slow heating process. If the barrier between the two ion structures is lower than the lowest energy dissociation barrier of the complex, a low amplitude heating period might allow for the conversion of some of the unreacted complex to the form in which the cycloaddition has occurred. A long-time, low-amplitude resonance excitation 'heating' experiment was used to test this idea. Figure 2(c) shows the CID spectrum of the [azide-K.K.KL/DIBACsufonate $+\mathrm{H}]^{+}$complex after 30 seconds of resonant excitation at an amplitude $24 \%$ of that of the CID voltage. The amplitude was selected to be just below the dissociation threshold on the 30 second time scale. After the low amplitude excitation period, another isolation was carried out in order to remove any minor fragments and the complex was subjected to the same CID conditions as those used for Figure 2(b). In the data of Figure 2(c), proton transfer gave rise to $5 \%$ of the product ion signal while backbone fragmentation gave rise to $95 \%$. The backbone fragmentation patterns in Figures 2(b) and 2(c) are essentially identical. The slow heating experiment therefore suggests that there is a mixture of structures present in the [azide-K.K.KL/DIBAC-sufonate $+\mathrm{H}]^{+}$complex 
population and that it is possible to increase the extent of cycloaddition by moderate heating of the ions.

The azide-K.K.KL/DIBAC-sulfonate species was also synthesized in solution for comparison with the gas-phase product. The solution phase coupled species was ionized in positive mode to predominantly yield the doubly protonated DIBAC-attached peptide. The singly protonated modified peptide was generated via a proton transfer ion/ion reaction with 2-iodo-5-methylbenzenesulfonate anions, then subsequently isolated and subjected to CID (Figure 2(d)). No proton transfer to yield the [azide-K.K.KL+H] ${ }^{+}$ion is observed which, in conjunction with Figures 2(b) and 2(c), confirms that proton transfer and backbone cleavage are not competing processes arising from a single ion/ion complex. The identities and relative abundances of the backbone fragments in Figures 2(b), 2(c), and 2(d), are very similar, indicating that the click chemistry products in both the solution and gas phases arise from the same cycloaddition reaction, albeit at varying primary amine sites within the peptide. 


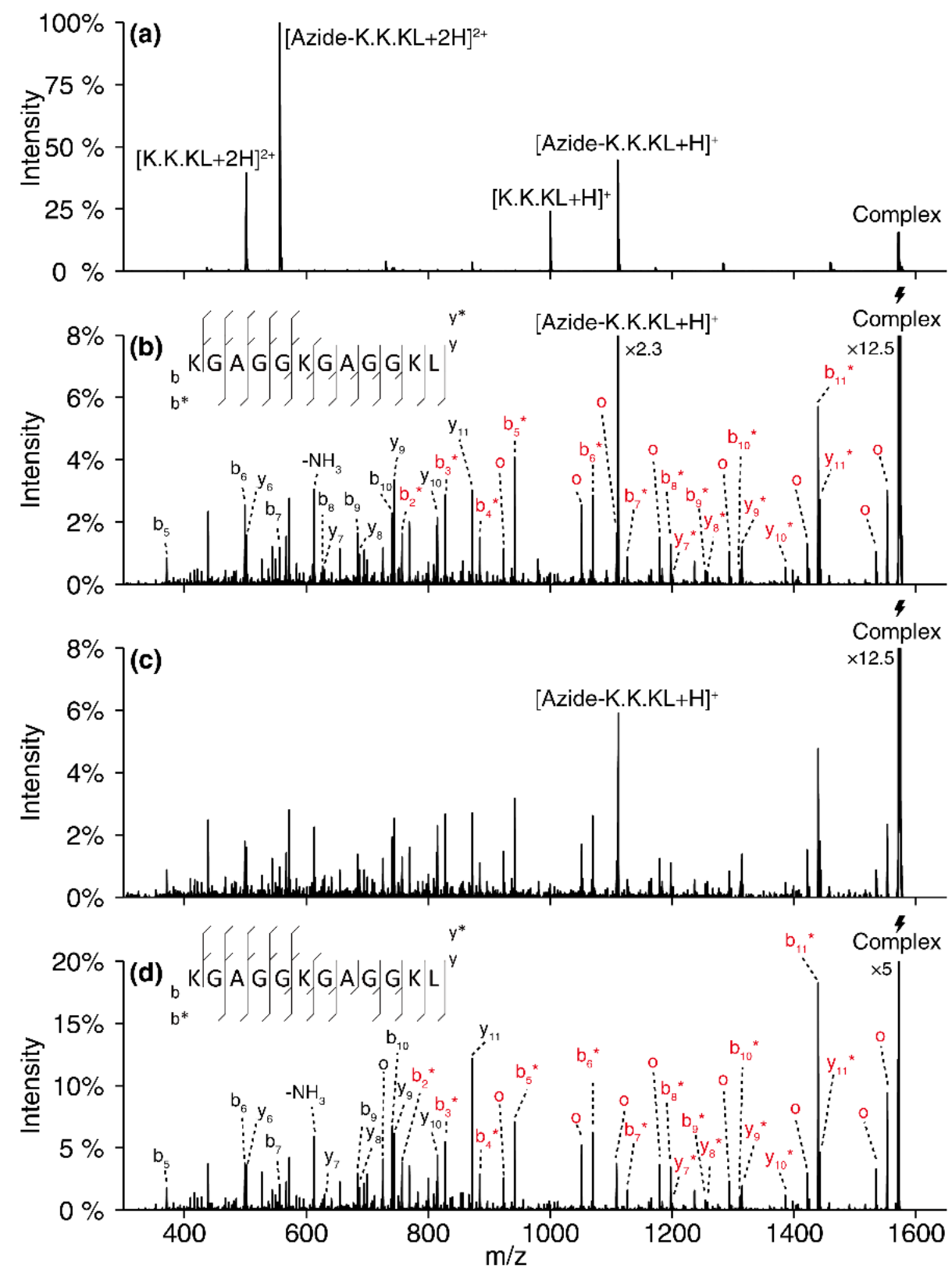

Figure 2. Spectra demonstrating (a) the gas phase ion/ion reaction between doubly protonated azide-K.K.KL and doubly protonated K.K.KL with DIBAC-sulfonate, (b) CID of the electrostatic complex comprized of azide-K.K.KL and DIBAC-sulfonate (indicated in the figure as 'complex'), (c) low amplitude resonance heating of the azideK.K.KL/DIBAC-sulfonate complex followed by CID, and (d) CID of the azideK.K.KL/DIBAC-sulfonate complex synthesized in solution. Degree signs $\left(^{\circ}\right)$ indicate water loss while red font indicates modified fragment ions. Modified b- and y-type fragment ions are also indicated with asterisks $(*)$. The lightning symbol $(\mathcal{N})$ is used to indicate the precursor subjected to CID. (KGAGGKGAGGKL $=$ K.K.KL) 
Click chemistry is also demonstrated with the generation of a negatively charged ion/ion complex, where Rhodamine 110-azide (Figure 1(d)), was generated by nESI in the positive mode as a singly protonated molecule $(\mathrm{m} / \mathrm{z}$ 575.2) and was allowed to react with doubly deprotonated DIBAC-disulfonate $(\mathrm{m} / \mathrm{z}$ 308.0) (Figure 1(c)). The attachment of the singly charged Rhodamine 110-azide cation to the dianion reagent results in a negatively charged complex $(\mathrm{m} / \mathrm{z}$ 1191.3) (Figure S1(c)). Activation of the isolated complex results in proton transfer from protonated Rhodamine 110-azide to doubly deprotonated DIBAC-disulfonate as well as loss of $\mathrm{SO}_{3}$ (f) from the complex (Figure 3(a)). Further isolation and $\mathrm{CID}$ of the $\mathrm{SO}_{3}$ loss product generates a series of fragments (Figure 3(c)), with a notable absence of the proton transfer product. A schematic diagram is included in Figure 3(d) to clarify the fragmentation patterns. The presence of fragments f4 and f1 in Figure 3(c) indicate that the click chemistry coupling occurs between the two cleaved amide bonds.

In analogy with the reaction between azide-K.K.KL and DIBAC-sulfonate described above, a long-time, low-amplitude resonance heating period for the [Rhodamine 110-azide/DIBAC disulfonate - H] $]^{-}$complex was employed prior to the CID step in the experiment leading to Figure 3(b). Specifically, the complex was isolated and subjected to a resonance heating at $12 \%$ of the CID voltage for 30 seconds. This amplitude was selected based on the same criteria described above. After the heating step, another isolation step was carried out in order to clear the ion trap of any minor fragments and the complex was subjected to CID for probing. The heating process clearly diminishes the proton transfer channel to the point where the $\mathrm{SO}_{3}$ loss process from the covalently modified species is predominant. 

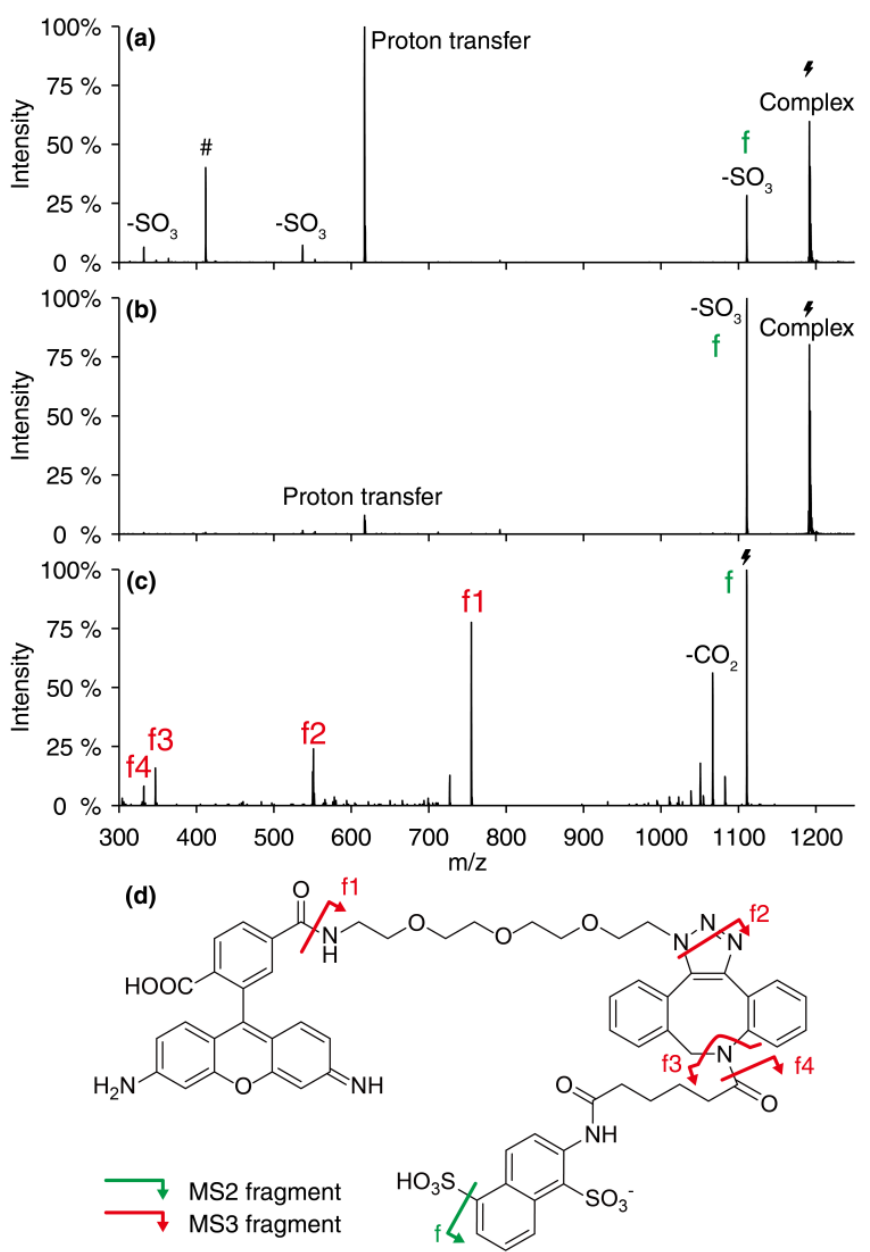

Figure 3. Spectra demonstrating (a) CID of the electrostatic complex generated via the gas phase reaction between protonated Rhodamine 110-azide and DIBAC-disulfonate, (b) low amplitude resonant heating of the complex followed by CID, (c) CID of the $\mathrm{SO}_{3}$ loss from the ion/ion reaction product complex. The scheme in (d) illustrates the fragmentation patterns in (c). The \# sign in (a) is the fragment corresponding to the $\mathrm{f} 4$ amide bond cleavage on a singly charged DIBAC-disulfonate reagent.

The Rhodamine 110-azide/DIBAC-disulfonate cycloaddition product was also synthesized in solution to compare with the gas-phase product. The solution-phase and gas-phase complexes follow the same fragmentation patterns upon activation, with the exception that less proton transfer product was observed for the gas-phase complex after the slow heating step (compare Figure 3(b) and Figure 4(a)). The leftover proton transfer product for the solution-phase synthesis may be due to incomplete reaction after 30 min 
resulting in the generation of some electrostatic complex upon nESI. The ionic products generated by the $\mathrm{SO}_{3}$ losses from activation of the solution-phase and gas-phase complexes were subjected to further CID. The spectra corresponding to activation of the $\mathrm{SO}_{3}$ loss products from the gas-phase (Figure 3(c)) and solution-phase (Figure 4(b)) are identical, further supporting the hypothesis that click chemistry cycloaddition can occur in the gas phase.

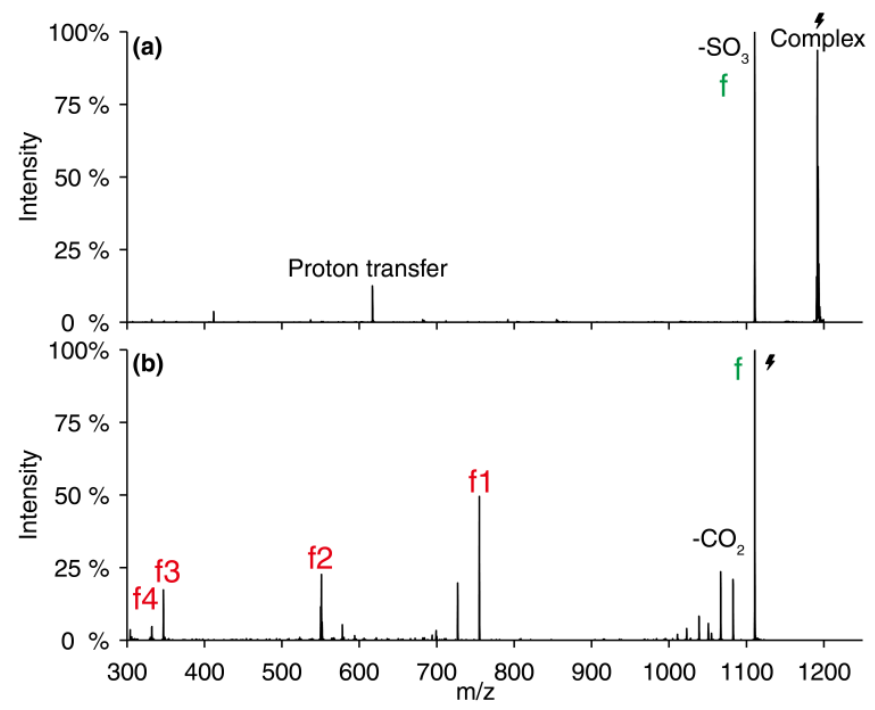

Figure 4. Spectra demonstrating (a) CID of the solution phase coupled complex between Rhodamine 110-azide and DIBAC-disulfonate, (b) CID of the $-\mathrm{SO}_{3}$ peak from the solution-phase complex.

DFT calculations using the Gaussian 09 package were performed on simple model systems to compare the energy requirements associated with the processes that can occur from the ion/ion complex. Upon formation, the complex is initially stabilized by the electrostatic interaction between a protonated site on the analyte ion and a sulfonate group on the reagent. The complex can subsequently undergo the cycloaddition reaction 'spontaneously' (i.e., in the absence of additional collisional heating) depending upon the energy surface associated with covalent reaction within the complex. The slow heating 
experiments leading to Figures 2(c) and 3(b) indicate that there is a significant barrier to the reaction that inhibits the reaction from proceeding with high efficiency in the absence of the slow heating step. Such an observation can be made only if the covalent reaction barrier can be overcome either before, or in the absence of, fragmentation. If there is a faster cleavage process at all energies, such as the proton transfer channel, little or no covalent reaction is expected. Figure 5 provides a generalized energy diagram that assigns zero as the energy of an electrostatically bound ion/ion complex. The diagram depicts the proton transfer channel to the left and the covalent reaction (i.e., rearrangement) channel to the right. Cleavage of covalent bonds from the electrostatic ion/ion complex is indicated as a possibility in gray trace to the right, although no clear indication for such cleavages was evident in the experiments associated with Figure 2. Minimal fragmentation of covalent bonds during the slow heating experiments also support the relative magnitudes of the fragmentation and cycloaddition barriers. The proton transfer exit channel was modeled by the proton transfer reaction between ethyl sulfonate and doubly protonated Tris(3-aminopropyl)methane to mimic the proton transfer barrier between azide-K.K.KL and DIBAC-sulfonate (see Supplementary Material for the coordinates of the calculated structures). The barrier for proton transfer was calculated to be $27.7 \mathrm{kcal} / \mathrm{mol}$. The click chemistry transition state (TS) barrier was modeled by the reaction between DIBAC-N-acetate and methyl azide. The TS barrier ZPE was determined to be $15.1 \mathrm{kcal} / \mathrm{mol}$. The TS for generic peptide backbone fragmentation has been estimated as $40 \mathrm{kcal} / \mathrm{mol}$ previously [40,41], which is consistent with the fact that fragments corresponding to this pathway were minimal in the slow heating experiments. Compared to the electrostatic complex, the click chemistry product 
is $62.2 \mathrm{kcal} / \mathrm{mol}$ lower in energy, which, at equilibrium, indicates that the click chemistry product is favored. The energetic requirements for peptide backbone cleavage of the click chemistry product are expected to be very similar to such cleavages from the electrostatic complex because the covalent modification in this case is not expected to impact any peptide cleavages. The results of the calculations are consistent with the experimental observations. While the barrier for covalent reaction is lower than that for proton transfer, a tighter transition state for the covalent reaction and possible steric constraints are expected to make the covalent reaction a relatively slow process. The slow heating steps used for the experiments of Figures 2(c) and 3(b) allow for more of the ion/ion complex structures to partition to the covalently modified form before the CID step.

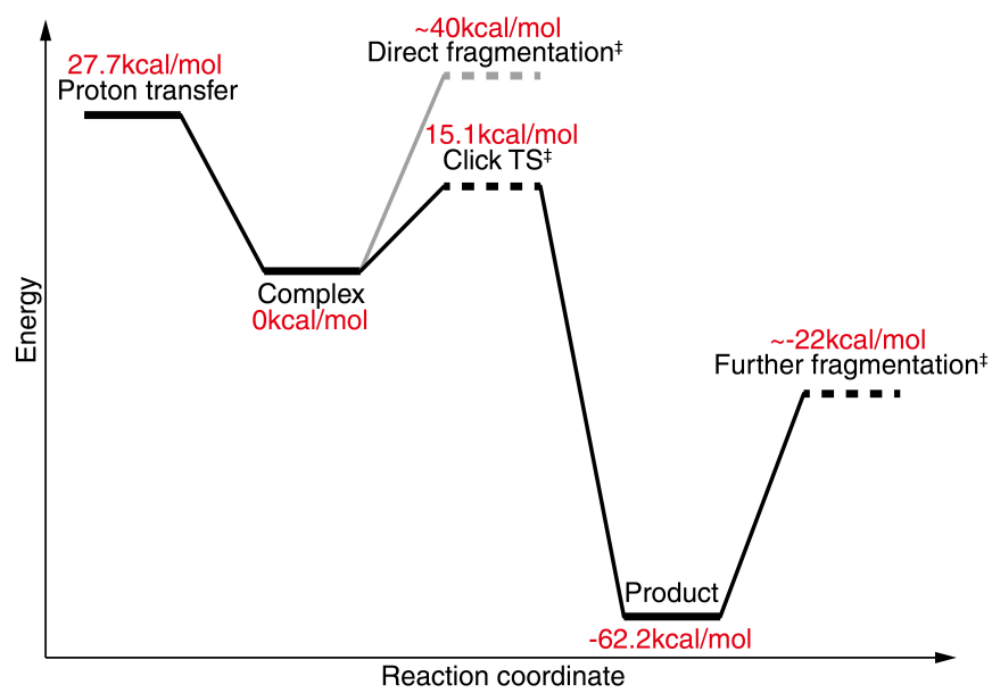

Figure 5. A reaction coordinate displaying the energies of reaction barriers and products of various pathways, relative to the starting ion/ion complex. Different pathways are modeled by different systems.

\section{CONCLUSIONS}


This work demonstrates the 1,3-dipolar cycloaddition reactivity of azide groups towards alkyne-containing DIBAC based reagents in the gas phase via ion/ion reactions. As with other gas-phase ion/ion reactions that result in covalent bond formation, the cycloaddition reaction proceeds via a long-lived ion/ion complex. The formation of a long-lived complex in the gas phase is facilitated by the presence of a sulfonate group on the reagent ion, which engages in a relatively strong interaction with a protonated site on the analyte. The strength of this interaction is important as it defines the barrier for proton transfer between the reactants, which establishes an upper limit for any barriers for covalent chemistry to be observed. Slow heating experiments indicate that a significant proportion of the initially formed ion/ion complex population does not cross the reaction barrier for covalent bond formation. However, a slow heating step can be used to drive unreacted complex ions to the covalently modified structure. DFT calculations of small model systems are consistent with the experimental data in that the barrier for cycloaddition is significantly lower than the barrier for proton transfer and fragmentation, and that the covalently-bound complex (i.e., the species generated from cycloaddition) is thermodynamically more stable than the electrostatically-bound complex. This work adds to the growing number of highly specific covalent reactions for analyte manipulation that can occur via gas-phase ion/ion chemistry.

\section{Acknowledgements}

The authors thank Zhe Dong from University of Texas at Austin for helpful discussions on reagent selection. This material is based upon work supported by the U.S. Department of Energy, Office of Science, Office of Basic Energy Sciences under Award Number FG02-00ER15105. 


\section{References}

[1] M. Dole, L.L. Mack, R.L. Hines, R. C. Mobley, L. D. Ferguson, M. B. Alice, Molecular Beams of Macroions. J. Chem. Phys., 49 (1968) 2240-2249.

[2] J.B. Fenn, M. Mann, C.K. Meng, S.F. Wong, C.M. Whitehouse, Electrospray ionization for mass spectrometry of large biomolecules. Science, 246 (1989) 64-71.

[3] K. Fujii, Y. Ikai, H. Oka, M. Suzuki, K. Harada, A nonempirical method using LC/MS for determination of the absolute configuration of constituent amino acids in a peptide: Combination of Marfey's method with mass spectrometry and its practical application. Anal. Chem., 69 (1997) 5146-5151.

[4] P.L. Ross, Y.L.N. Huang, J.N. Marchese, B. Williamson, K. Parker, S. Hattan, N. Khainovski, S. Pillai, S. Dey, S. Daniels, S. Purkayastha, P. Juhasz, S. Martin, M. Bartlet-Jones, F. He, A. Jacobson, D.J. Pappin, Multiplexed protein quantitation in Saccharomyces cerevisiae using amine-reactive isobaric tagging reagents. Mol. Cell. Proteomics, 3 (2004) 1154-1169.

[5] S. Gygi, B. Rist, S. Gerber, F. Turecek, M. Gelb, R. Aebersold, Quantitative analysis of complex protein mixtures using isotope-coded affinity tags. Nat. Biotechnol., 17 (1999) 994-999.

[6] J. Madsen, J. Brodbelt, Simplifying Fragmentation Patterns of Multiply Charged Peptides by N-Terminal Derivatization and Electron Transfer Collision Activated Dissociation. Anal. Chem., 81 (2009) 3645-3653.

[7] R.L. Beardsley, L.A. Sharon, J.P. Reilly, Peptide de Novo Sequencing Facilitated by a Dual-Labeling Strategy. Anal. Chem., 77 (2005) 6300-6309.

[8] W. Yang, H. Mirzaei, X. Liu; F.E. Regnier, Enhancement of Amino Acid Detection and Quantification by Electrospray Ionization Mass Spectrometry. Anal. Chem., 78 (2006) 4702-4708.

[9] B.M. Prentice, S.A. McLuckey, Gas-phase ion/ion reactions of peptides and proteins: acid/base, redox, and covalent chemistries. Chem. Commun., 49 (2013) 947-965.

[10] S.A. McLuckey, M. Mentinova, Ion/Neutral, Ion/Electron, Ion/Photon, and Ion/Ion Interactions in Tandem Mass Spectrometry: Do We Need Them All? Are They Enough? J. Am. Soc. Mass Spectrom., 22 (2011) 3-12.

[11] S. Osburn, V. Ryzhov, Ion-Molecule Reactions: Analytical and Structural Tool. Anal. Chem., 85 (2013) 769-778.

[12] H.P. Gunawardena, M. He, P.A. Chrisman, S.J. Pitteri, J.M. Hogan, B.D.M. Hodges, S.A. McLuckey, Electron transfer versus proton transfer in gas-phase ion/ion reactions of polyprotonated peptides. J. Am. Chem. Soc., 127 (2005) 12627-12639.

[13] J.E.P. Syka, J.J. Coon, M.J. Schroeder, J. Shabanowitz, D.F. Hunt, Peptide and protein sequence analysis by electron transfer dissociation mass spectrometry. Proc. Natl. Acad. Sci. U.S.A., 101 (2004) 9528-9533.

[14] W.J. Herron, D.E. Goeringer, S.A. McLuckey, Product ion charge state determination via ion/ion proton transfer reactions. Anal. Chem., 68 (1996) 257-262.

[15] S.A. McLuckey, The emerging role of ion/ion reactions in biological mass spectrometry: considerations for reagent ion selection. Eur. J. Mass Spectrom., 16 (2010) 429-436. 
[16] K.M. Hassell, J.R. Stutzman, S.A. McLuckey, Gas-Phase Bioconjugation of Peptides via Ion/Ion Charge Inversion: Schiff Base Formation on the Conversion of Cations to Anions. Anal. Chem., 82 (2010) 1594-1597.

[17] M. Mentinova, S.A. McLuckey, Covalent Modification of Gaseous Peptide Ions with N-Hydroxysuccinimide Ester Reagent Ions. J. Am. Chem. Soc., 132 (2010) 18248-18257.

[18] W.M. McGee, M. Mentinova, S.A. McLuckey, Gas-Phase Conjugation to Arginine Residues in Polypeptide Ions via N-Hydroxysuccinimide Ester-Based Reagent Ions. J. Am. Chem. Soc., 134 (2012) 11412-11414.

[19] Z. Peng, W.M. McGee, J.X. Bu, N.Z. Barefoot, S.A. McLuckey, Gas Phase Reactivity of Carboxylates with N-Hydroxysuccinimide Esters. J. Am. Soc. Mass Spectrom., 26 (2015) 174-180.

[20] B.M. Prentice, J.D. Gilbert, J.R. Stutzman, W.P. Forrest, S.A. McLuckey, Gas-Phase Reactivity of Carboxylic Acid Functional Groups with Carbodiimides. J. Am. Soc. Mass Spectrom., 24 (2013) 30-37.

[21] A.L. Pilo, S.A. McLuckey, Oxidation of Methionine Residues in Polypeptide Ions Via Gas-Phase Ion/Ion Chemistry. J. Am. Soc. Mass Spectrom., 25 (2014) 10491057.

[22] A.L. Pilo, J. Bu, S.A. McLuckey, Generation of $[\mathrm{M}-\mathrm{H}]+,[\mathrm{M}+\mathrm{H}+\mathrm{O}]+$, and $\mathrm{M}+\bullet$ Peptide Cations via Ion/Ion Reactions with a Suite of Reagents Derived from Persulfate. J. Am. Soc. Mass Spectrom., (In press) DOI: 10.1007/s13361-015-1125-y

[23] J. Gilbert, B. Prentice, S. McLuckey, Ion/Ion Reactions with "Onium” Reagents: An Approach for the Gas-phase Transfer of Organic Cations to Multiply-Charged Anions. J. Am. Soc. Mass Spectrom., (2015) 1-8.

[24] H.C. Kolb, M.G. Finn, K.B. Sharpless, Click chemistry: Diverse chemical function from a few good reactions. Angew. Chem. Int. Ed., 40 (2001) 2004-2021.

[25] R. Huisgen, Centenary Lecture - 1,3-Dipolar Cycloadditions. Proc. Chem. Soc., (1961), 357-396.

[26] V.V. Rostovtsev, L.G. Green, V.V. Fokin, K.B. Sharpless, A stepwise Huisgen cycloaddition process: Copper(I)-catalyzed regioselective "ligation" of azides and terminal alkynes. Angew. Chem., 114 (2002) 2708-2711.

[27] C.W. Tornoe, C. Christensen, M. Meldal, Peptidotriazoles on solid phase: [1,2,3]triazoles by regiospecific copper(I)-catalyzed 1,3-dipolar cycloadditions of terminal alkynes to azides. J. Org. Chem., 67 (2002) 3057-3064.

[28] J.M. Baskin, J.A. Prescher, S.T. Laughlin, N.J. Agard, P.V. Chang, I.A. Miller, A. Lo, J.A. Codelli, C.R. Bertozzi, Copper-free click chemistry for dynamic in vivo imaging. Proc. Natl. Acad. Sci. U.S.A., 104 (2007) 16793-16797.

[29] X.H. Ning, J. Guo, M.A. Wolfert, G.J. Boons, Visualizing metabolically labeled glycoconjugates of living cells by copper-free and fast huisgen cycloadditions. Angew. Chem. Int. Ed., 47 (2008) 2253-2255.

[30] M.F. Debets, S.S. van Berkel, S. Schoffelen, F.P.J.T. Rutjes, J.C.M. van Hest, F.L. van Delft, Aza-dibenzocyclooctynes for fast and efficient enzyme PEGylation via copper-free (3+2) cycloaddition. Chem. Commun., 46 (2010) 97-99. 
[31] J.C. Jewett, E.M. Sletten, C.R. Bertozzi, Rapid Cu-Free Click Chemistry with Readily Synthesized Biarylazacyclooctynones. J. Am. Chem. Soc., 132 (2010) 36883690 .

[32] K. Nwe, M.W. Brechbiel, Growing Applications of "Click Chemistry" for Bioconjugation in Contemporary Biomedical Research. Cancer Biother. Radiopharm., 24 (2009) 289-302.

[33] G.T. Hermanson, BioconjugateTechniques, Academic Press/Elsevier, Amsterdam (2013).

[34] J.W. Hager, A new linear ion trap mass spectrometer. Rapid Commun. Mass Spectrom., 16 (2002) 512-526.

[35] Y. Xia, X.R. Liang, S.A. McLuckey, Pulsed dual electrospray ionization for ion/ion reactions. J. Am. Soc. Mass Spectrom., 16 (2005) 1750-1756.

[36] X. Xia, W. Jin, S.A. McLuckey, F.A. Londry, J.W. Hager, Mutual storage mode ion/ion reactions in a hybrid linear ion trap. J. Am. Soc. Mass Spectrom., 16 (2005) 71-81.

[37] F.A. Londry, J.W. Hager, Mass selective axial ion ejection from a linear quadrupole ion trap. J. Am. Soc. Mass Spectrom., 14 (2003) 1130-1147.

[38] M.J. Frisch, G.W. Trucks, H.B. Schlegel, G.E. Scuseria, M.A. Robb, J.R. Cheeseman, G. Scalmani, V. Barone, B. Mennucci, G.A. Petersson, H. Nakatsuji, M. Caricato, X. Li, H.P. Hratchian, A.F. Izmaylov, J. Bloino, G. Zheng, J.L. Sonnenberg, M. Hada, M. Ehara, K. Toyota, R. Fukuda, J. Hasegawa, M. Ishida, T. Nakajima, Y. Honda, O. Kitao, H. Nakai, T. Vreven, J.A. Montgomery Jr., J.E. Peralta, F. Ogliaro, M.J. Bearpark, J. Heyd, E.N. Brothers, K.N. Kudin, V.N. Staroverov, R. Kobayashi, J. Normand, K. Raghavachari, A.P. Rendell, J.C. Burant, S.S. Iyengar, J. Tomasi, M. Cossi, N. Rega, N.J. Millam, M. Klene, J.E. Knox, J.B. Cross, V. Bakken, C. Adamo, J. Jaramillo, R. Gomperts, R.E. Stratmann, O. Yazyev, A.J. Austin, R. Cammi, C. Pomelli, J.W. Ochterski, R.L. Martin, K. Morokuma, V.G. Zakrzewski, G.A. Voth, P. Salvador, J.J. Dannenberg, S. Dapprich, A.D. Daniels, Ö. Farkas, J.B. Foresman, J.V. Ortiz, J. Cioslowski, D.J. Fox, Gaussian 09, Gaussian, Inc., Wallingford, CT, 2009.

[39] K. Fukui, The path of chemical-reactions - The IRC approach. Acc. Chem. Res., 14 (1981) 363-68.

[40] B. Paizs, S. Suhai, Towards understanding the tandem mass spectra of protonated oligopeptides. 1: Mechanism of amide bond cleavage. J. Am. Soc. Mass Spectrom., 15 (2004) 103-113.

[41] I.P. Csonka, B. Paizs, G. Lendvay, S. Suhai, Proton mobility and main fragmentation pathways of protonated lysylglycine. Rapid Commun. Mass Spectrom., 15 (2001) 1457-1472. 